\title{
Evidence Based Approach for the Diagnosis of Temporomandibular Joint Disorders (TMD)
}

\author{
Muralidhar Mupparapu
}

Published online: 30 August 2013

(C) Indian Prosthodontic Society 2013

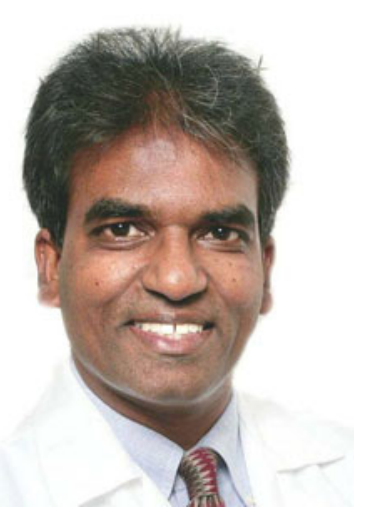

Temporomandibular disorders comprise a unique group of pathological entities that have complex etiopathogenesis and are extremely difficult to identify early on in the course of the disease even with the sophisticated imaging protocols that are currently available. The clinician is often left with history and clinical findings only before getting a chance to investigate the findings further. It is therefore, quite interesting to see what the current evidence is that has been universally accepted for the diagnosis of temporomandibular joint disorders (TMD).

It is fair to say that the research diagnostic criteria for temporomandibular disorders (RDC/TMD) that are universally accepted for the clinical and radiographic diagnosis of TMD use very stringent guidelines for the determination of various disorders that are related to TMD. Ahmad et al. [1] detailed in their paper the criteria for image analysis for diagnosis of TMJ osteoarthritis. They

M. Mupparapu ( $\square)$

University of Pennsylvania School of Dental Medicine,

Philadelphia, PA 19104, USA

e-mail: mmupparapu@gmail.com categorized the criteria into three groups- A, B and C. They have grouped those with relatively normal size of the condylar head without any subcortical sclerosis or surface flattening, no known subcortical cystic changes, no surface erosions and no evidence of osteophytosis or generalized sclerosis into group A. Similarly, they have grouped those patients with or without articular flattening, with or without subcortical sclerosis into group B. Finally, only those with clear deformation within the condyles due to subcortical cyst, surface erosions, osteophytosis or generalized sclerosis were classified into the category $\mathrm{C}$. Now, if we ponder further into the rigor with which these are categorized, one would realize quickly that often, the group A or group B category of patients may actually be in the initial phases of TMD where there were no obvious radiographic findings. The clinical picture more frequently reflecting one of the following: myofascial pain, disc related pain, occlusal discrepancy or pain that appears to be neuropathic in origin. In all of these situations, there would be very little morphological changes to the TM joint. How do we then go about diagnosing these TMD patients?

Understanding the fundamental mechanisms involved in the liberation of inflammatory byproducts or those that are released in response to pain have changed the way we understand the TMD. Attempts have been made to utilize the biomarkers of inflammation and bone turnover for early diagnosis of various TMDs. Evidence should be gathered to identify all of the byproducts of inflammation, pain and tissue destruction related to the TMJ. At the same time, the evidence should be gathered to make sure that these byproducts that are liberated are very specific to the affliction of TMJ and are uniquely produced as a result of the TMJ-related pathoses. Literature shows that this is the most daunting task in the field of TMD that both basic scientists and clinicians are faced with. Large scale 
population based studies have isolated the "at-risk" groups for the TMD but the "lack of early diagnostic indicators" have made these studies rather uninteresting. Further, we know that the patients who seek help for TMD are a minority of the larger group of patients who may have the TMD but are not severely symptomatic forcing them seek help. Quite often, the typical TMD patient seeking help is in an advanced form of TMD that is identifiable on radiographs and clinically symptomatic. Although, the subjects with TMD are managed via both conservative and surgical means, the latent period of the disease process remains an enigma.

Let us look at the serological or tissue based evidence that we can gather in an attempt to reach an early diagnosis of TMD. The categories that were investigated dealt with either the presence or absence of:

1. Mediators of inflammation such as interleukins (IL) or cytokines

2. Neuropeptides like substance $\mathrm{P}$ or calcium generelated peptide(CGRP)

3. Matrix metallo-proteinases

4. Inhibitors of tissue inflammatory mediators

TMJ internal derangements and osteoarthritis are known to be associated with inflammatory processes in the synovial membrane and articular cartilage [2]. The cytokines that are released secondary to the inflammation within the joint such as IL-1, IL-6, TNF-alpha can potentially cause cartilage degradation through upregulation of metalloproteinases gene expression and a decrease in the chondrocyte compensatory synthesis pathways. There are substances that are expressed secondary to nociception like CGRP and substance $P$ have been investigated by various researchers. The TMJ-related structures are mostly covered with fibrous rather than hyaline cartilage unlike the majority of the joints in the body. Fibrocartilage contains mostly type I collagen and the hyaline cartilage contains mostly type II collagen. Type I collagen accounts for over $90 \%$ of organic part of the bone. An increased concentration of CTx-I, the C-terminal cross-linked telepeptides of collagen type I indicates loss of both TMJ fibrocartilage and bone. So, this is not a specific indicator. Similarly, the increased levels of CTx-II can only indicate loss of hyaline cartilage via degenerative or inflammatory changes in the body [3]. This will not help the attempts to diagnose TMD. Hence additional specific tests must be considered. Attempts were made to use pyridinoline and deoxypyridinoline as markers for the diagnosis of TMD. Since these markers are present in the TMJ cartilage and they are not metabolized in the body, their elimination via kidneys would be a good clinical test to determine the degree of TMD. Although the investigators could find a good correlation between the increased levels of Pyr and dPyr in the urine that was detected via high performance liquid Chromatography, no correlation was found to their increased levels and radiographic features suggestive of TMD. Similarly, plasma bradykinin has been investigated to see if the marker is a specific diagnostic marker for TMD [3]. Even though the serum levels correspond to those of synovial fluid levels, it has never been used as a diagnostic or prognostic indicator. We now know that specific information regarding the TMD especially if it is inflammatory in origin can be obtained from biochemical examination of the synovial fluid and that their levels correspond with the joint pain, all three aspects of analysis including bone turnover markers, assessment of inflammatory markers and imaging appear to be the only way one could accurately diagnose either degenerative changes or inflammatory processes affecting the TMJ. Future TMD research should be directed at specific evidence-based mechanisms for both diagnosis and management of TMD.

\section{References}

1. Ahmad M, Hollander L, Anderson Q, Kartha K, Ohrbach R, Truelove EL et al (2009) Research diagnostic criteria for temporomandibular disorders (RDC/TMD): development of image analysis criteria and examiner reliability for image analysis. Oral Surg Oral Med Oral Pathol Oral Radiol Endod 107:844-860

2. Fernandes JC, Martel-Pelletier J, Pelletier JP (2002) The role of cytokines in osteoarthritis pathophysiology. Biorheology 39:237-246

3. Kostrzewa-janicka J, Mierzwinska-Natalskaur E, Jurkowski P, Okonski P, Nedzi-Gora M (2013) Assessment of temporomandibular disease. neurobiology of respiration. In: Pokorski M (ed) Advances in experimental medicine and biology. Springer science, Dordrecht, pp 207-211 